\title{
Los recreos cooperativos: una metodología de y para la socialización Cooperative playgrounds: a methology for the socialization
}

\author{
Ana Ma Mega Avellaneda, Cecilia Latorre Cosculluela \\ 647793@unizar.es, cecilialacos@gmail.com
}

\author{
${ }^{1}$ Ciencias de la Educación \\ Facultad de Ciencias Humanas y de la Educación \\ Huesca, España
}

\author{
${ }^{2}$ Ciencias de la Educación \\ Facultad de Ciencias Humanas y de la Educación \\ Huesca, España
}

\begin{abstract}
Resumen Esta comunicación presenta un estudio que se ha llevado a cabo con niños/as de Educación Primaria de dos centros educativos públicos de Huesca; C.E.I.P. Pio XII y C.E.I.P. Pedro J. Rubio. La intención es explorar cuáles son los efectos de un proyecto de aprendizaje cooperativo desarrollado en los recreos de dos centros de Educación Infantil y Primaria diferenciados por el nivel sociocultural de la población. Se pretende conocer si los valores emocionales que subyacen de la práctica lúdica contribuyen a eliminar situaciones de exclusión social, y si los efectos del juego cooperativo, difieren o no, entre dos realidades que representan a la diversidad de la ciudad. Los resultados manifiestan como el alumnado, independientemente del contexto del que proviene, mejora sus habilidades intra e interpersonales, y por ende se generan relaciones más positivas.
\end{abstract}

\section{Palabras clave: Conductas prosociales, inclusión, juego cooperativo} y comunicación.

Abstract This communication presents a study that has been carried out with children of Primary Education in two state schools in Huesca; C.E.I.P. Pío XII and C.E.I.P. Pedro J. Rubio. The intention is to explore what effects of a cooperative learning project are. The aim is to discover whether learning resulting is differentiated by the socio-cultural level of the population, and if emotional values of playing contribute to eliminate situations of social exclusion of two realities that represent the diversity of the city. The results show how students, regardless of the context that comes, improve their intra and interpersonal skills, and how cooperative games cause more positive educational relationships.

Keywords: Prosocial behavior, inclusion, cooperative play and Communication.

\section{INTRODUCCIÓN}

El aumento de las situaciones de acoso escolar, en todas sus manifestaciones, junto a los programas socioemocionales que están resultando ser insuficientes en la adquisición de conductas prosociales, parece ser un tema que preocupa a la comunidad educativa, pues hay rivalidades, ocultas y explícitas durante el recreo, que interfieren negativamente en la convivencia escolar y por ende, en el bienestar emocional (Garaigordobil y Fagoaga, 2006).

Si echamos una mirada, sin necesidad de adentrarnos en las aulas, observamos alumnos aislados, acosados y/o ridiculizados durante el recreo. ¿Por qué, un espacio que implica libertad y diversión, deja de ser el momento esperado para algunos alumnos? Esta problemática nos lleva a pensar que, todavía, existe la necesidad de seguir creando programas educativos que promuevan, desde tempranas edades, la interculturalidad, y estimulen el desarrollo de la competencia socioemocional, pues de éstas dependerá el autoconcepto y las habilidades inter e intrapersonales que dinamizan las relaciones positivas, y en base a las que se forja la personalidad de un alumno (Goleman y Davidson, 2012).

Algunos autores manifiestan que es necesario dotar a los alumnos de unas habilidades comunicativas y sociales que promuevan la “cooperación” y fomenten la participación y la socialización (Pujolás, 2013), porque las habilidades sociales no son innatas al ser humano, y además, éstas son necesarias para trabajar en grupo y convivir en la diversidad; autoconcepto, comunicación interpersonal, percepciones, gestión de las emociones y sentimientos, capacidad de crítica, cooperación, ayuda, empatía, resolución de conflictos, y flexibilización (Álvarez, 2011 y Camacho, 2013).

En esta línea, podemos pensar que la cooperación podría ser una estrategia inclusiva, contraria a la competitividad, a desarrollar en los recreos, y encaminada a educar bajo la ayuda incondicional, la solidaridad entre los iguales y la aceptación (Pujolás, 2003). Así, nuestro estudio pretende valorar si el aprendizaje resultante de las interacciones cooperativas difiere, o no, de un contexto educativo a otro, si sirve para evitar situaciones conflictivas durante el recreo escolar, y si mejora la discriminación de género entre chicas y chicos en los momentos de juego en ambos centros.

\section{CONTEXTO}

La unidad de análisis corresponde al proyecto de "Recreos Cooperativos e Inclusivos”, una experiencia de la Facultad de Ciencias Humanas y de la Educación de Huesca. En el estudio participan dos centros públicos de Huesca con realidades sociales y culturales diferentes entre sí que representan, en buena parte, a la diversidad presente en nuestra ciudad.

Por un lado, el alumnado de $3^{\circ}$ y $4^{\circ}$ de E.P del C.E.I.P. Pedro J. Rubio; un colegio deseado por las familias, con un exceso 
de demanda y una elevada ratio, situado en un entorno sociodemográfico con estabilidad laboral, y con un nivel socioeconómico medio-alto. Por otro lado, el alumnado de $1^{\circ}$ y $2^{\circ}$ de E.P del C.E.I.P. Pio XII; un contexto que aglutina a gran parte de la población en riesgo de exclusión social y en situaciones desfavorables; mayoritariamente población de etnia gitana y/o de origen inmigrante que, además, cuenta con dos aulas de Educación Especial.

La elección de la muestra se ha confeccionado a partir de la decisión que toma el equipo directivo de ambos centros, seleccionando estos cursos y no otros, por la presencia en ellos, de grupos sociales en situaciones de riesgo social o exclusión. En ambos centros educativos encontramos alumnado asilado durante el recreo, comportamientos agresivos y/o disruptivos, algún grupo cultural minoritario, e incluso algún caso de acoso escolar.

\section{DESCRIPCIÓN}

Con el fin de dar respuesta a los objetivos del estudio se ha diseñado un marco metodológico descriptivo, pues se pretende analizar qué emociones suscita el juego cooperativo, y las vivencias de éstas en relación con las conductas observadas en los momentos de juego.

La finalidad del proyecto de "Recreos Cooperativos" es contribuir a la mejora de las habilidades sociales del alumnado y la convivencia escolar. Este estudio propone, en primer lugar, valorar si el aprendizaje resultante del juego cooperativo difiere, o no, de unos contextos educativos u otros; en segundo lugar, conocer si el juego mejora la relación social entre chicos y chicas en el recreo; y finalmente, identificar si el juego cooperativo sirve para reducir las situaciones conflictivas presentes durante los recreos.

La experiencia se llevó a cabo durante tres meses a razón de una sesión semanal de juegos en la hora del recreo, cuyas sesiones fueron planificadas por los estudiantes de Magisterio en prácticas, tras recibir una formación inicial por la Ludoteca Municipal de Huesca. Con el fin de operativizar la recogida y el análisis de datos sistematizamos el proceso en varias fases.

- Fase I. Realización de una prueba piloto formada por una pequeña muestra de ocho alumnos elegidos al azar de entre el grupo de la muestra participante.

- Fase II. Elaboración de la hoja de observación con las conductas comportamentales observables e identificables en la prueba piloto.

- Fase III. Validez y fiabilidad de estos instrumentos, por varios jueces expertos.

- Fase III. Creación de grupos base cooperativos y asignación de un estudiante de Magisterio/tutor a cada uno de estos.

- Fase IV. Recogida de información del alumnado de Magisterio (registro observacional).

- Fase V. Contrastar información en reuniones periódicas de coordinación con el EOEIP.

- Fase VI. Recogida de información del alumnado de Educación Primaria (prueba colectiva a partir de un cuestionario).

- Fase VII. Análisis de los resultados obtenidos y valoración de la experiencia.
Para la recogida de los datos se utiliza un cuestionario de evaluación destinado al alumnado partícipe, de cada uno de los centros, y una hoja de observación para el profesorado, coordinador, y alumnos de prácticas de Magisterio.

La validación de contenido se garantizó presentando ambos instrumentos de evaluación a 5 jueces expertos de la Universidad de Zaragoza, quienes contaban con una pauta para evaluar de 1 a 5 el análisis de la congruencia y pertinencia de los ítems al propósito del cuestionario, así como un espacio para proponer cambios y/o sugerencias. En el cuestionario se modificaron algunos ítems por no superar el índice acordado entre los jueces.

Para determinar la fiabilidad o consistencia interna del cuestionario, se llevó a cabo una prueba piloto con 4 alumnos elegidos al azar de entre la muestra participante de ambos centros. Los resultados arrojaron un coeficiente de Alfa de Cronbach de 0,87, el cual excede el valor mínimo de 0,70 para determinar la confiabilidad de la prueba (DeVellis, 2003; Kline, 2000).

La versión final del cuestionario consta de siete ítems de tipo Likert de respuestas alternativas y con un espacio para que el alumnado añada información adicional. Esta prueba colectiva se ha pasado a un total de 111 alumnos. En la tabla 1 se sintetiza la estructura del cuestionario y la información que se recoge en relación con los objetivos del estudio.

Tabla 1. Variables recogidas en la prueba de evaluación colectiva aplicada al alumnado.

\begin{tabular}{cl} 
Relación & \multicolumn{1}{c}{ Variables } \\
social & $\begin{array}{l}\text { Entre chicas y chicos. } \\
\text { Percepción de mis compañeros. } \\
\text { Autopercepción respecto al grupo. }\end{array}$ \\
Conciencia & Vivencia de emociones compartidas. \\
emocional & $\begin{array}{l}\text { Autoconcepto y bienestar al jugar. } \\
\text { Autorregulación en otros ámbitos. }\end{array}$ \\
& $\begin{array}{l}\text { Interdependencia positiva. } \\
\text { Celebración del éxito grupal. } \\
\text { Cooperación } \\
\end{array}$ \\
& $\begin{array}{l}\text { Resolución de conflictos. } \\
\text { Habilidades interpersonales. }\end{array}$
\end{tabular}

La versión final de la hoja de observación destinada al profesorado recoge dos ítem; el comportamiento del alumnado (conductas problemáticas, relaciones interpersonales e implicación); una valoración de los juegos programados y el éxito de la planificación, posibles mejoras, con una puntuación entre 0 y 5 (la más alta). Además se incluyen 4 preguntas abiertas para recoger información sobre dificultades encontradas, medidas correctoras, aspectos positivos y previsiones para la semana siguiente.

\section{Resultados}

Uno de los objetivos era conocer si el juego cooperativo servía para mejorar las relaciones sociales entre chicos y chicas, y si era posible compartir intereses comunes.

En ambos centros se observaban situaciones discriminatorias de género que repercutían en el apego y en los lazos afectivos 
entre chicos y chicas, en un marco de expectativas y profecía negativa, que perjudicaba a las relaciones sociales y que ocasionaban situaciones de exclusión, aislamiento y peleas entre grupos.

Los comportamientos y las actitudes mostradas según el sexo, parecían ser aspectos de retraimiento a la hora de jugar todos juntos, pues las chicas destacaban por ser más sedentarias, pasivas, tímidas e inseguras, y los chicos por presentar un carácter más competitivo, mostrándose reacios a jugar en equipos mixtos.

Los resultados indican que el nivel de interdependencia de los grupos cooperativos mixtos alcanza, al finalizar el proyecto, un rango porcentual muy positivo en ambos centros; un $12 \%$ y $23 \%$ puntúan la relación entre chicos y chicas como "regular"; un 82\% y $65 \%$ como "buena"; y sorprendentemente, un 95\% y 79,4 \% como "muy buena”, en el C.E.I.P. Pio XII y C.E.I.P. Pedro J. Rubio, respectivamente. Las mejoras son evidentes, pues el aprender a relacionarse desde el juego cooperativo ha supuesto una mejora de las relaciones interpersonales y la adquisición de valores como la igualdad de género, el respeto, la convivencia escolar, el liderazgo y el bienestar personal.

$\mathrm{Al}$ analizar las respuestas que ofrece el alumnado sobre lo que siente al jugar cooperativamente, se refleja una mejora en la convivencia de ambos centros. Los resultados muestran una correlación inversa entre los estereotipos “conflictivos y problemáticos” de un centro y las conductas prosociales que muestran. Así, el 53 \% del alumnado del C.E.I.P. Pío XII y el 22.22 \% del C.E.I.P. Pedro J. Rubio “temían las posibles represalias si su equipo fracasaba por su culpa”. Sin embargo, estos comportamientos competitivos se van convirtiendo en cooperativos conforme transcurren las sesiones.

Al finalizar el proyecto las valoraciones positivas indicadas por el alumnado difieren, también, de un centro a otro. Así, un 88,3 \% del alumnado del C.E.I.P. Pío XII, frente a un 57,14 \% del alumnado del C.E.I.P. Pedro J. Rubio consideran que "todos son necesarios para lograr el éxito". Sorprendentemente, el centro con mayores rivalidades culturales obtiene mejores puntuaciones e incluso aparecen, con mayor frecuencia, gestos de apoyo y de ánimo recíprocos. A diferencia de ello, en el C.E.I.P Pedro J. Rubio surgen más dificultades para configurar grupos cooperativos; únicamente el 19,05 \% “se siente más aceptado", y el 17,45 \% "se siente mejor consigo mismo” a diferencia del C.E.I.P. Pio XII donde los porcentajes alcanzados son mayores respectivamente; $70,6 \%$ y un $53 \%$.

$\mathrm{Al}$ valorar las relaciones interpersonales y las competencias emocionales que les suscita el juego cooperativo, se obtienen puntuaciones muy positivas, pues el 94,9\% de la muestra total indica "sentirse emocionado y feliz al jugar con sus compañeros/as”, argumentando que "ahora dialogamos en la resolución de conflictos”, "respetamos la opinión de los compañeros” y “nos expresamos sin miedo”. En definitiva, son ellos mismos quienes remarcan los principios en los que se fundamenta la cooperación.

En relación a las habilidades interpersonales que muestran durante el juego, los alumnos de menor edad (CEIP Pio XII) tienden a ofrecer mayor "ayuda mutua” (un $88 \%$ frente a un 57,14\%), “confianza” (88,3\% frente a un $59 \%$ ), y “perdonar y agradecer” más fácilmente ya que tienen menos prejuicios hacia sus iguales (96\% frente a un 62\%).

Se observa como conforme van madurando, la imagen que se crean de sí mismos es más negativa. Así, solo un 48,3 \% del alumnado del C.E.I.P. Pedro J. Rubio ( $3^{\circ}$ y $4^{\circ}$ E.P) “se acepta tal y como es”, en contraposición, el alumnado del C.E.I.P. Pío XII ( $1^{\circ}$ y $2^{\circ}$ E.P.) que alcanza mejor puntuación (75,4 \%).

Estos datos parecen indicar la importancia de crear experiencias cooperativas, desde tempranas edades ya que precisamos alumnos capaces de proyectar lo bueno, en lugar de enjuiciar y hundir su autoestima.

Otro de los objetivos era valorar si la cooperación servía de premisa para disminuir conflictos sociales y aumentar estilos de conductas asertivas.

Los resultados indican que el alumnado del C.E.I.P. Pío XII considera más positivamente al ítem de la comunicación. Así, un $96 \%$ del alumnado, considera primordial dialogar para solucionar los conflictos, aunque realmente no es capaz de hacerlo sin un mediador, frente a un 75,9\% del alumnado del C.E.I.P Pedro J. Rubio. Esta diferencia puede verse marcada por el programa de "Medicación Escolar" puesto en marcha en el C.E.I.P. Pío XII. En dicho centro, el alumnado de quinto y sexto recibe formación específica sobre habilidades implicadas en la resolución pacífica, y de entre estos, se seleccionan a los mediadores/as. Estos, cuando surgen conflictos, median entre los alumnos en conflicto, quienes acuden a un aula destinada exclusivamente para ello.

Las rivalidades, observadas por el profesorado, se dan mayoritariamente entre alumnado de diferentes etnias o cultura. Este hecho enfatiza la necesidad de trabajar las rivalidades sociales y extrapolar esa confianza y ayuda entre la diversidad presente en los centros.

Finalmente, la valoración general de los agentes educativos implicados en el proyecto (profesorado, orientador, estudiantes de Magisterio y coordinador) ponen de manifiesto que cualquier juego que erradique conductas competitivas, individualistas o sexistas, es útil para estimular una educación que valora al juego como algo positivo, porque permite el desarrollo de personas solidarias, comprometidas y respetuosas con sus iguales. No existe un juego mejor que otro, sino que un juego será bueno, siempre y cuando, se ajuste a las necesidades sociales del contexto.

\section{CONCLUSIONES}

Una vez analizada la información recogida mediante diferentes instrumentos, se presentan las conclusiones donde se confrontan los datos obtenidos del estudio.

En nuestros inicios apostamos por la práctica de dinámicas comunicativas, cooperativas y lúdicas con el fin de optimizar las habilidades sociales del alumnado, y mejorar así, la convivencia escolar. Indudablemente, las rivalidades sociales, guetos culturales o situaciones de discriminación y exclusión, que tenían lugar en los recreos, han disminuido 
considerablemente. Coincidimos con Pujolás (2013) en que el juego cooperativo ofrece una educación cívica y moral que permite la adquisición de valores y habilidades sociales al requerir de la participación activa de todos los miembros de un grupo cooperativo.

Otro de nuestros interrogantes era la eficacia que el juego cooperativo podía tener para erradicar conductas destructoras, agresivas o inapropiadas. En este sentido los resultados son muy esperanzadores y coinciden con los resultados de otros estudios similares (Cerdas, 2013; Garaigordobil 2007; Valeiras e Iturriaga, 2008), pues ha servido para que los alumnos adquieran conductas prosociales, y mejoren las relaciones entre chicas y chicos.

A través de los juegos cooperativos se ha conseguido reconducir comportamientos que evidenciaban la interrelación social pobre, los conflictos sociales, la indiferencia o los escasos lazos afectivos entre el alumnado de índole étnica diversa.

Resulta importante señalar que el proyecto de Recreos Cooperativos, en sus diferentes manifestaciones, es una experiencia que toma como referencia las interacciones sociales como fuente de aprendizaje emocional. Es más, las consecuencias de propiciar una educación emocional a través del juego se refleja en los resultados, donde más del 80 \% del alumnado manifiesta que han aprendido a valorarse $\mathrm{y}$, aceptarse tal y como son, porque se sienten más seguros consigo mismo y con menos temor a actuar por el miedo a equivocarse. Observamos cómo el alumno, al adquirir habilidades intrapersonales, es capaz de interactuar más fácilmente, porque respetan la importancia de la comunicación y las diferentes perspectivas, siendo ellos quienes, argumentan la importancia de confiar en los demás, responsabilizarse de uno mismo y por el grupo, y estar unidos para vencer.

Finalmente, encontramos que el aprendizaje resultante de las interacciones cooperativas difiere de un contexto educativo a otro. Sorprendentemente, los resultados obtenidos en el C.E.I.P. Pío XII han sido más favorables que en el C.E.I.P. Pedro J. Rubio, pues alcanzan mejores valores relativos a la igualdad de sexos, el respeto, la ayuda mutua y el bienestar emocional y social.

Una vez más las condiciones socioculturales desmienten la adversidad que se tiende a creer de que en centros con contextos más desfavorecidos, no es posible lograr una educación de calidad. Situaciones como éstas son las que enjuician el prestigio de centros educativos como el C.E.I.P. Pío XII, el cual se ve afectado por estereotipos equívocos relacionados con la calidad de la educación que ofrece (Ainscow, 2012; Dueñas, 2010; Escudero, 2012; Echeita, 2013), y que por las condiciones desfavorecidas de su alumnado parten de una clara desventaja o riesgo de generarse situaciones de exclusión. Sin embargo, a pesar de existir un estereotipo muy extendido respecto a los gitanos e inmigrantes, según el cual se les atribuye indiscriminadamente una serie de mensajes segregadores, de acuerdo con Abadajo (2004), la imagen que se crea de ellos no coincide siempre con los resultados.
Concluimos afirmando que el proyecto de jugar cooperativamente encamina al alumnado a vivir experiencias que le permiten valorar cuáles son las emociones y los sentimientos que nos hacen sentir bien o mal a las personas, y actuar en base a la empatía. Recordamos que los niños son el reflejo y el futuro de nuestra sociedad, de manera que si pretendemos que ellos muestren comportamientos que no devalúen a sus iguales, desde las escuelas se han de promover proyectos que defiendan este tipo de valores.

Por lo tanto, el proyecto de Recreos Cooperativos e Inclusivos compromete al alumnado a alejarse de actitudes competitivas y segregadoras. Si los conflictos sociales y las actitudes de desconfianza se gestionan correctamente y no son vistos como un problema sino como una oportunidad, podemos lograr el cambio de actitudes en escuelas que indagan en la cultura del diálogo y la cooperación.

En definitiva, el juego cooperativo es capaz de erradicar prejuicios fijados socialmente porque no presionan psicológicamente, sino que rompen con la rivalidad y "nadie es mejor que nadie”. En este sentido, al crear ambientes más relajados, el juego, desde la bonanza de la diversión, impregna la necesidad de buscar estrategias y soluciones conjuntas. Y, así, el alumnado inconscientemente adquiere valores de afirmación, escucha, comunicación, creatividad, e integración.

\section{REFERENCIAS}

Abadajo, J.E. (2004). Infancia gitana y paya: Convivencia y conflictos en la escuela. Tabanque: Revista pedagógica, 18, 97-116.

Ainscow, M. (2012). Haciendo que las escuelas sean más inclusivas: lecciones a partir del análisis de la investigación internacional. Revista de Educación inclusiva, 5(1), 39-49.

Álvarez, D.G. (2011). La Inteligencia Emocional: competencias esenciales para la vida ciudadana. REDIELUZ, 1(1), 72-79.

Camacho, L.J. (2013). El juego cooperativo como promotor de habilidades sociales en niñas de 5 años (Tesis de pregrado). Pontificia Universidad Católica del Perú, Perú.

Cerdas, E. (2013). Experiencias y aprendizajes con juegos cooperativos. Instituto de estudios Latinoamericano, 6, 107-123.

DeVellis, R.F. (2003). Scale development: Theory and applications ( $2^{\mathrm{a}}$ ed.). Newbury Park, CA: Sage.

Dueñas, M ${ }^{\mathrm{a}}$ L. (2010). Educación inclusiva. REOP, 21(2), 358-366.

Echeita, G. (2013). Inclusión y Exclusión Educativa. De Nuevo, “Voz y Quebranto". REICE. Revista Iberoamericana sobre Calidad, Eficacia y Cambio en Educación, 11(2), 99-118.

Escudero, J.M. (2012). La educación inclusiva, una cuestión de derecho. Educatio Siglo XXI, 2, 109-128

Garaigordobil, M. (2007). Intervención psicoeducativa para el desarrollo de la Personalidad Infantil: Los programas JUEGO. (Conferencia de Clausura). Congreso Internacional de Orientación Educativa y Profesional: "Nuevos enfoques educativos y su repercusión en la orientación escolar. Castellón. Universitat Jaume I. 
Garaigordobil, M. y Fagoaga, J.M. (2006). Juego cooperativo para prevenir la situación de los escolares. Madrid: Ministerio de Educación y Ciencia.

Goleman, D. y Davidson, R. (2012). Aprender a gestionar las emociones. Programa de Redes. Recuperado de: http://www.rtve.es/televisión/20121025/aprendergestionar-emociones/571611.shtml

Kline, P. (2000). The handbook of psychological testing $\left(2^{\mathrm{a}}\right.$ ed). New York: Routlege.

Pujolás, P. (2003). Aulas inclusivas y aprendizaje cooperativo. Educatio Siglo XXI, 30(1), 89-112.

Pujolás, P. (2013). El aprendizaje cooperativo. 9 ideas clave. Barcelona: Graó.
Valeiras, J. A. y Iturriaga, M. A. (2008). Utilización del recreo escolar por niños de $4^{\circ}$ y $6^{\circ}$ de Primaria. Retos: nuevas tendencias en educación física, deporte y recreación, 14, 88-91. 